\title{
Posttranslational modification and mutation of histidine 50 trigger alpha synuclein aggregation and toxicity
}

Wei Xiang ${ }^{1 * \dagger}$, Stefanie Menges ${ }^{2+}$, Johannes CM Schlachetzki ${ }^{2}$, Holger Meixner ${ }^{2}$, Anna-Carin Hoffmann ${ }^{1}$, Ursula Schlötzer-Schrehardt ${ }^{3}$, Cord-Michael Becker', Jürgen Winkler ${ }^{2}$ and Jochen Klucken ${ }^{2}$

\begin{abstract}
Background: Aggregation and aggregation-mediated formation of toxic alpha synuclein (aSyn) species have been linked to the pathogenesis of sporadic and monogenic Parkinson's disease (PD). A novel H50Q mutation of aSyn, resulting in the substitution of histidine by glutamine, has recently been identified in PD patients. We have previously shown that the lipid peroxidation product 4-hydroxy-2-nonenal (HNE) induces the formation of HNE-aSyn adducts, thereby promoting aSyn oligomerization and increasing its extracellular toxicity to human dopaminergic neurons. Intriguingly, we identified histidine 50 (H50) of aSyn as one of the HNE modification target residues. These converging lines of evidence support the hypothesis that changes in $\mathrm{H} 50$ via posttranslational modification (PTM) and mutation trigger the formation of aggregated, toxic aSyn species, which interfere with cellular homeostasis. In the present study, we aim to elucidate 1) the role of H50 in HNE-mediated aSyn aggregation and toxicity, and 2) the impact of H50 mutation on aSyn pathology. Besides the PD-related H50Q, we analyze a PD-unrelated control mutation, in which H50 is replaced by an arginine residue (H50R).

Results: Analysis of HNE-treated aSyn revealed that H50 is the most susceptible residue of aSyn to HNE modification and is crucial for HNE-mediated aSyn oligomerization. Overexpression of aSyn with substituted $\mathrm{H} 50$ in $\mathrm{H} 4$ neuroglioma cells reduced HNE-induced cell damage, indicating a pivotal role of H5O in HNE modification-induced aSyn toxicity. Furthermore, we showed in vitro that $\mathrm{H50Q} / \mathrm{R}$ mutations substantially increase the formation of high density and fibrillar aSyn species, and potentiate the oligomerization propensity of aSyn in the presence of a nitrating agent. Cell-based experiments also revealed that overexpression of H50Q aSyn in H4 cells promotes aSyn oligomerization. Importantly, overexpression of both $\mathrm{H} 50 \mathrm{Q} / \mathrm{R}$ aSyn mutants in $\mathrm{H} 4$ cells significantly increased cell death when compared to wild type aSyn. This increase in cell death was further exacerbated by the application of $\mathrm{H}_{2} \mathrm{O}_{2}$.
\end{abstract}

Conclusion: A dual approach addressing alterations of $\mathrm{H} 50$ showed that either H50 PTM or mutation trigger aSyn aggregation and toxicity, suggesting an important role of aSyn $\mathrm{H} 50$ in the pathogenesis of both sporadic and monogenic PD.

Keywords: Parkinson's disease, Alpha synuclein, 4-hydroxy-2-nonenal, Posttranslational modification, Histidine 50, H50Q mutation

\footnotetext{
* Correspondence: wei.xiang@fau.de

${ }^{\dagger}$ Equal contributors

${ }^{1}$ Institute of Biochemistry (Emil-Fischer-Center), Friedrich-Alexander-University

of Erlangen-Nürnberg (FAU), Fahrstraße 17, 91054 Erlangen, Germany
Full list of author information is available at the end of the article

of Erlangen-Nürnberg (FAU), Fahrstraße 17, 91054 Erlangen, Germ
Full list of author information is available at the end of the article
}

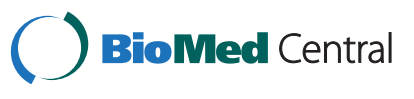

(c) 2015 Xiang et al.; licensee BioMed Central. This is an Open Access article distributed under the terms of the Creative Commons Attribution License (http://creativecommons.org/licenses/by/4.0), which permits unrestricted use, distribution, and reproduction in any medium, provided the original work is properly credited. The Creative Commons Public Domain Dedication waiver (http://creativecommons.org/publicdomain/zero/1.0/) applies to the data made available in this article, unless otherwise stated. 


\section{Background}

Parkinson's disease (PD) is the most prevalent neurodegenerative movement disorder worldwide. The disease is associated with a progressive loss of dopaminergic neurons in the substantia nigra pars compacta. An important neuropathological hallmark is the formation of eosinophilic inclusions called Lewy bodies (LB) and Lewy neurites in brains of PD patients, mainly consisting of aggregated alpha synuclein (aSyn), along with other proteins such as neurofilament subunits and ubiquitin [1]. The etiology of PD has not been fully understood. Environmental factors and genetic variants, which are proposed to affect neuronal function and eventually lead to degenerative processes, have been linked to an increased risk for PD. In particular, molecular and cellular disturbances including oxidative stress, mitochondrial impairment, and abnormal protein processing have been closely associated with PD [2].

Although the majority of PD cases are of unknown (sporadic) etiology, distinct genetic mutations of different genes account for approximately $10 \%$ of PD cases [3]. In particular, five point mutations in the coding sequence of the aSyn gene (SNCA) have been linked to autosomal dominant inherited PD, including the intensively studied A53T, A30P, and E46K mutations [4-6], and the very recently discovered $\mathrm{H} 50 \mathrm{Q}$ and G51D mutations [7-9]. In addition, duplication and triplication of SNCA have been identified in familial forms of PD [10-12], suggesting that overexpression of the aSyn protein disturbs neuronal homeostasis. Furthermore, genome wide association studies revealed that genetic variants of SNCA are closely linked to sporadic PD $[13,14]$. The pathological relevance of aSyn in PD is further supported by the observation that aggregated and posttranslational modified aSyn is the major protein component in LB [15]. All these lines of evidence support a crucial role of aSyn, particularly of its aggregated forms, in the pathogenesis of PD.

Physiologically, aSyn is a soluble protein and is predominantly localized in neurons at presynaptic terminals in close proximity to synaptic vesicles [16]. aSyn aggregation is a process, in which the physiological soluble aSyn proteins change in conformation to produce pathological aggregated forms. Aggregated aSyn species include oligomers and fibrils, characterized by increased beta sheet content, insolubility, and detergent resistance [17]. Yet, it has not been clarified which factors trigger aggregation of aSyn and which aggregated aSyn species interfere with neuronal homeostasis in PD pathogenesis. Accumulating evidence supports that oxidative stress is an important modifier of aSyn aggregation. Oxidative stress is characterized by the accumulation of reactive oxygen/nitrogen species (ROS/RNS), which affect biomolecules, leading to posttranslational modifications (PTMs) of proteins and lipid peroxidation. Some of the generated aldehyde products during lipid peroxidation/degradation, such as 4-hydroxy-2-nonenal (HNE), are highly reactive to proteins and further trigger PTMs by forming aldehyde adducts on target proteins [18]. We have recently shown that ROS/RNS and HNE modify aSyn and promote the aggregation of aSyn [19]. Importantly, HNE modification of aSyn enhances the neurotoxicity of aSyn $[20,21]$, in particular to human dopaminergic neurons [19]. Using mass spectrometry, we identified histidine 50 (H50) of aSyn as one of the target residues of HNE modification. Interestingly, a novel SNCA mutation, resulting in an amino acid exchange of $\mathrm{H} 50$ with a glutamine residue (H50Q), was recently reported in two PD patients [7,9]. These converging lines of evidence suggest that alterations of H50, via either PTM or genetic mutation, influence the aggregation propensity of aSyn and eventually induce cell damage.

To elucidate the relevance of H50 for aSyn pathology, in particular for aSyn aggregation and cytotoxicity, we investigated the role of H50 in 1) HNE-mediated, and 2) H50 mutation-induced aSyn pathology. In addition to $\mathrm{H} 50 \mathrm{Q}$, we analyzed a PD-unrelated mutation in order to answer the question of whether the potential changes induced by the PD-related $\mathrm{H} 50 \mathrm{Q}$ mutation are due to the loss of $\mathrm{H} 50$, or are attributed to the specific effect of the glutamine residue. To this end, we replaced $\mathrm{H} 50$ by an arginine residue (H50R), which belongs to the group of polar and basic amino acids like histidine. Our results show that both posttranslational and genetic modifications of aSyn at the specific H50 residue increase the aggregation propensity and toxicity of aSyn.

\section{Results}

aSyn $\mathrm{H} 50$ is the most reactive residue for HNE modification HNE, a lipid peroxidation product, reacts with histidine, cysteine, and lysine residues of proteins, leading to the formation of HNE-protein adducts [18]. Since aSyn does not have cysteine residues, the only histidine residue (H50) and 15 lysine residues are potential targets for HNE modification. Recently, we showed that $\mathrm{H} 50$ of aSyn is a target residue for HNE modification, although it is not the only possible modification site in aSyn [19]. Indeed, HNE modifications of Lys 60 and Lys 96 have also been described [22]. Thus, our first aim was to elucidate the role of H50 for HNE modification in aSyn.

We applied site-directed mutagenesis to exchange the $\mathrm{H} 50$ residue and to produce two recombinant aSyn H50 mutants, i.e. H50Q and H50R. HNE concentration ranges in vivo from $0.1-3 \mu \mathrm{M}$ under physiological conditions and may increase up to $10-5000 \mu \mathrm{M}$ under pathological conditions of increased oxidative stress [18,23]. In order to analyze the reactivity of $\mathrm{H} 50$ to $\mathrm{HNE}$, we incubated recombinant wild type (WT) and $\mathrm{H} 50 \mathrm{Q} / \mathrm{R}$ mutant aSyn with pathologically relevant HNE concentrations $(50-3000 \mu \mathrm{M})$. The addition of one HNE molecule 
to a target amino acid residue is characterized by a mass increase of $156 \mathrm{Da}$. Matrix-assisted laser-desorptionionization time-of-flight mass spectrometry (MALDI-TOF MS) analysis of GluC-digested WT aSyn exposed to HNE revealed HNE modification of the $\mathrm{H} 50$ containing peptide ${ }^{47}$ GVVHGVATVAE $^{57}$ (Figure 1A). The shift from unmodified (*) to modified peptide $(\downarrow)$ increased in a HNE concentration-dependent manner. Both $\mathrm{H} 50$ mutants (H50Q/R) completely abolished HNE modification of the corresponding residue 50 containing peptides (Figure 1B).

MALDI-TOF MS analysis of full-length aSyn revealed that HNE addition to WT aSyn is already detectable at a HNE concentration of $50 \mu \mathrm{M}$ (Figure 1C). Incubation of WT aSyn with HNE at low concentrations $(50-200 \mu \mathrm{M})$ resulted in the addition of a single HNE molecule. HNE concentrations from 500 to $3000 \mu \mathrm{M}$ induced the formation of additional HNE adducts in WT aSyn, indicating the existence of more than one modifiable amino acid residue at high HNE concentrations. In contrast to WT aSyn, HNE adducts were barely detectable in aSyn H50 mutants (H50Q/R) exposed to low HNE concentrations (50 - $200 \mu \mathrm{M}$ ). Only high concentrations of HNE applied to H50 mutant aSyn led to the formation of HNE adducts. This result revealed that other modifiable residues of aSyn (e.g. lysine residues) exhibit a lower reactivity to HNE and thus indicate that $\mathrm{H} 50$ is the initial target residue of $\mathrm{HNE}$ modification.

\section{aSyn $\mathrm{H} 50$ is the crucial residue for HNE-mediated oligomerization}

HNE triggers the oligomerization of aSyn [19]. As lysine residues may also be involved in HNE modification, we asked whether HNE modification of $\mathrm{H} 50$ is the major factor for HNE-mediated oligomerization. We exposed recombinant human WT and $\mathrm{H} 50 \mathrm{Q} / \mathrm{R}$ aSyn to different HNE concentrations and investigated the HNE-mediated oligomerization by SDS-PAGE followed by Western blot (WB) analysis (Figure 2A and B) and size exclusion chromatography (SEC) (Figure $2 \mathrm{C}$ and $\mathrm{D}$ ). We observed that SDS-resistance of aSyn oligomers is dependent on the applied HNE concentration. SDS-resistant oligomers of WT aSyn were detectable after exposure to $3 \mathrm{mM}$ HNE (Figure 2A). Importantly, at the same HNE concentration, i.e. $3 \mathrm{mM}, \mathrm{H} 50$ mutations reduced the formation of HNE-induced, SDS-resistant aSyn oligomers (Figure 2B). This effect was reproducibly detected using different antibodies raised against distinct aSyn antigens. Using SEC analysis, we observed an increase in the formation of soluble WT aSyn oligomers after HNE exposure (Figure 2C). In accordance to the results of
A

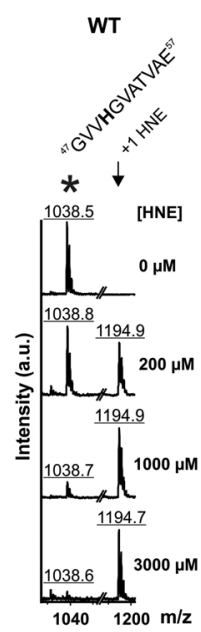

B

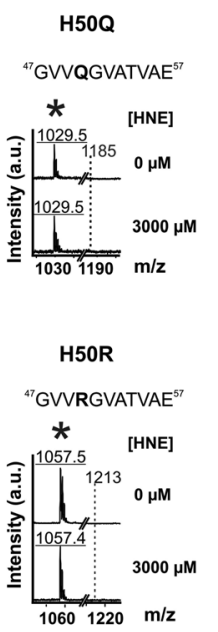

C

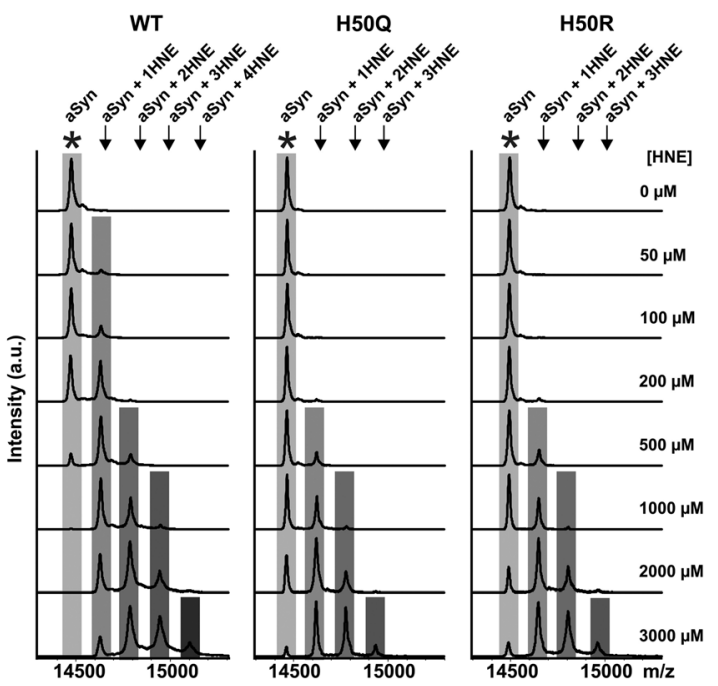

Figure 1 HNE modification of WT and H50 mutant aSyn. A) Recombinant WT aSyn treated with different concentrations of HNE $(0-3000 \mu M)$ for $24 \mathrm{~h}$ was digested by GluC in order to measure HNE modification of the $\mathrm{H} 50$ containing peptide ${ }^{47} \mathrm{GWHGVATVAE} \mathrm{E}^{57}$ by MALDI-TOF analysis. The modification of this peptide (*observed monoisotopic mass to charge ratio m/z: 1038) by HNE leads to a mass increase of 156 Da ( $\downarrow$ observed monoisotopic m/z: 1194). B) aSyn H50Q and H50R mutations completely abolish HNE modification of the corresponding peptides containing the residue 50. Only unmodified peptides, i.e. ${ }^{47}$ GWQGVATVAE ${ }^{57}$ ( ${ }^{*}$ observed monoisotopic m/z: 1029) of the H50Q aSyn and ${ }^{47}$ GWRGVATVAE ${ }^{57}$ ( ${ }^{*}$ observed monoisotopic m/z: 1057) of the H5OR aSyn, are observed. C) Recombinant full-length WT and H50Q/R aSyn were treated with different HNE concentrations for $24 \mathrm{~h}$ and analyzed by MALDI-TOF MS. Unmodified $(*)$ and HNE-modified $(\downarrow)$ aSyn (WT, H50Q, and H50R) with a characteristic mass difference of $156 \mathrm{Da}$ are indicated. H50Q/R mutations reduce the formation of aSyn-HNE-adducts, particularly they prevent the modification by HNE at low concentrations $(50-200 \mu \mathrm{M})$. 


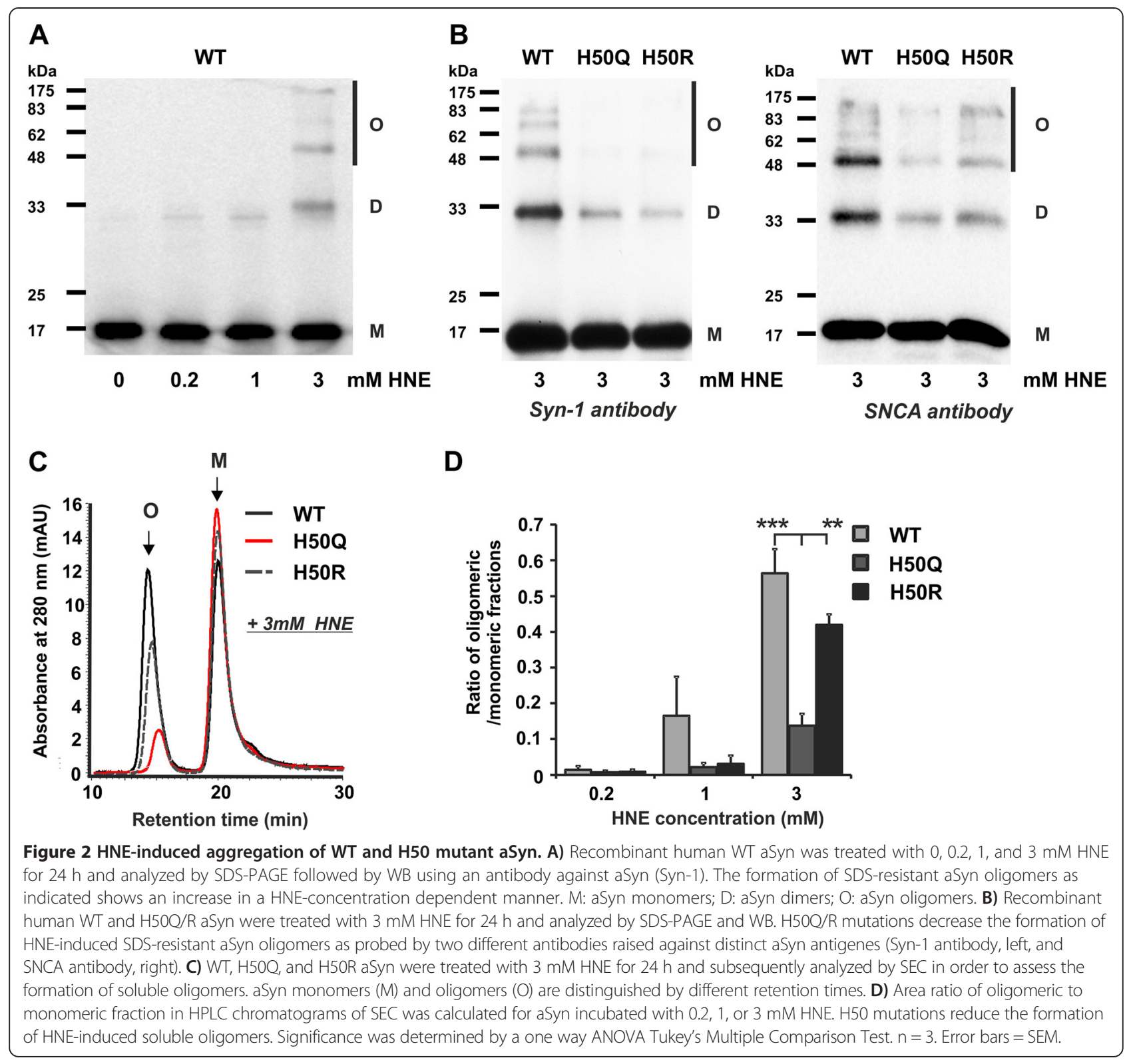

SDS-PAGE, H50 mutations, in particular H50Q, also decreased the formation of HNE-mediated soluble oligomers (Figure 2D). While SDS-PAGE detects SDSresistant oligomers (both soluble and insoluble), SEC examines soluble oligomers (both SDS-resistant and non-resistant). Both approaches provided consistent results, indicating that $\mathrm{H} 50$ is the initial factor contributing to HNE-induced oligomerization of aSyn.

\section{The substitution of aSyn $\mathrm{H} 50$ reduces HNE-induced cytotoxicity}

HNE modification increases the cytotoxicity of aSyn when applied to cells extracellularly [19]. Furthermore, HNE modification of intracellularly overexpressed aSyn was shown in cells exposed to extracellular HNE [22].
Thus, we asked whether $\mathrm{H} 50$, the most reactive target residue of aSyn for HNE modification (as described above, Figure 1), influences the susceptibility of aSyn overexpressing cells to HNE exposure. To address this question, we generated pcDNA3.1 plasmids encoding human WT or H50Q/R mutant aSyn for transient transfection in mammalian cells. To reduce the interference between endogenous and overexpressed aSyn variants, we chose human $\mathrm{H} 4$ neuroglioma cells, which only express very low levels of endogenous aSyn. aSyn overexpression was confirmed via immunocytochemistry (ICC) and flow cytometric analysis (Figure 3A, B and C). The proportion of aSyn transfected cells showed a time dependent increase, reaching 39 - 48\% $36 \mathrm{~h}$ after transfection (Additional file 1: Figure S1). H4 cells transfected 


\section{A}
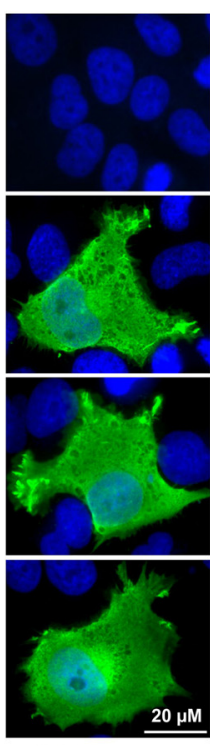

DAPI aSyn

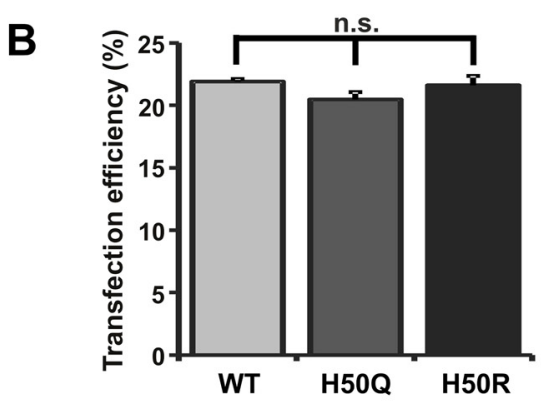

C

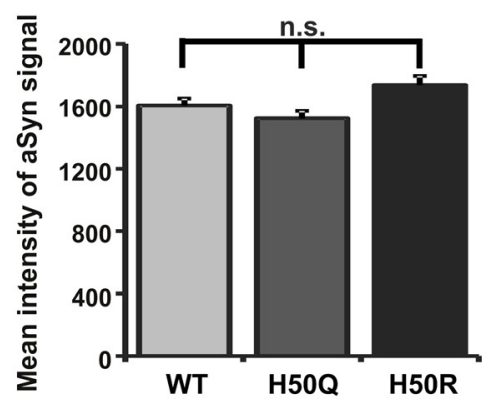

\section{D}

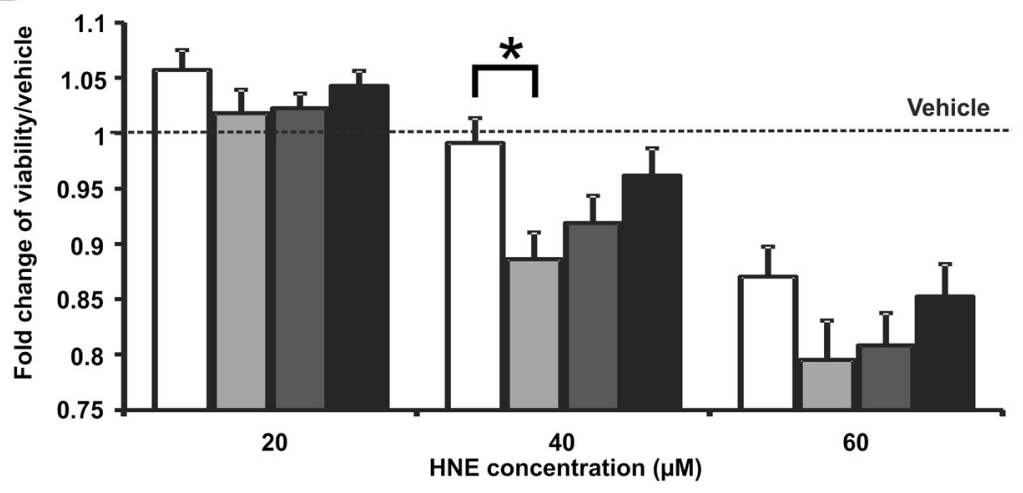

Figure 3 HNE-induced cytotoxicity in WT and H50 mutant aSyn overexpressing cells. A) Expression of aSyn in H4 cells transiently transfected with WT aSyn and H50 mutants (H50Q/R) was confirmed by ICC using an antibody against human aSyn (green) and counterstaining by DAPI (blue). B) Transfection efficiency and C) aSyn expression levels in $\mathrm{H} 4$ cells transfected with WT aSyn and H50 mutants were assessed via flow cytometry at the time point of HNE treatment (24 h posttransfection). WT and H50Q/R aSyn transfected cells show an equal proportion of aSyn overexpressing cells (B) as well as comparable aSyn expression levels (C). Significance was determined by one way ANOVA Tukey's Multiple Comparison Tests. $n=3$. Error bars $=S E M$. D) H4 cells transiently transfected with mock, WT, and H50Q/R aSyn were treated with different HNE concentrations $(20-60 \mu \mathrm{M})$ for $12 \mathrm{~h}$ and cellular viability was measured by MTS assay. HNE induces a dose-dependent reduction of cell viability. H50Q/R mutant aSyn transfected cells appear to be more resistant to HNE than WT aSyn transfected cells. Significance was determined by two way ANOVA Bonferroni Multiple Comparisons. $n=8$. Error bars $=S E M$.

with WT, H50Q, and H50R aSyn vectors showed a comparable transfection rate for all measured time points $(17-36 \mathrm{~h}$ posttransfection, Additional file 1: Figure S1C). No significant differences in both transfection efficiency and aSyn expression levels were detected $24 \mathrm{~h}$ after transfection, when we started the treatment experiments (Figure $3 \mathrm{~B}$ and $\mathrm{C}$ ). We incubated WT and H50Q/R mutant aSyn, as well as mock transfected cells, with different concentrations of HNE and measured cell damage by using MTS viability assay. While HNE exposed
WT aSyn overexpressing cells showed a reduced viability compared to mock-transfected cells for the HNE concentrations used $(20-60 \mu \mathrm{M})$, in particular for $40 \mu \mathrm{M} \mathrm{HNE}$, cell damage of H50Q and H50R overexpressing H4 cells exposed to the same HNE concentrations was less pronounced (Figure 3D). The complementary flow cytometric analysis of transfected $\mathrm{H} 4$ cells treated with HNE supported that H50 mutant aSyn overexpressing cells, in particular the aSyn H50R overexpressing cells, are more resistant to HNE exposure than WT aSyn transfected cells 
(Additional file 1: Figure S2). These results suggest that the exchange of the $\mathrm{H} 50$ residue attenuates the toxic effect of $\mathrm{HNE}$, supporting the essential role of $\mathrm{H} 50 \mathrm{HNE}$ modification for HNE-induced cytotoxicity.

\section{$\mathrm{H} 50$ mutations increase the aggregation propensity of} aSyn both in vitro and in cells

Modification of H50 is crucial for HNE-induced aSyn oligomerization. Thus, we asked whether the alteration of $\mathrm{H} 50$ through genetic point mutation, particularly the novel reported $\mathrm{H} 50 \mathrm{Q}$ mutation, also influences the aggregation propensity of aSyn. In addition to $\mathrm{H} 50 \mathrm{Q}$, we analyzed a non PD-related control mutation (H50R) to specify whether changes caused by $\mathrm{H} 50 \mathrm{Q}$ mutation are due to the loss of $\mathrm{H} 50$ or attributed to the specific effect of the glutamine residue.

To evaluate the intrinsic aggregation propensity of mutant $\mathrm{H} 50$ variants, we applied sucrose density gradient centrifugation (SDGC), and used the collected fractions for dot blot analysis in order to detect aggregation of aSyn. To optimize the separation range of the density gradient, we first analyzed control samples including WT monomeric aSyn (WT, Figure 4, upper panel), highly oligomerized HNE-modified WT aSyn (HNE-aSyn, Figure 4 lower panel), nitrated WT aSyn (n-aSyn, Figure 4 lower panel) as characterized previously [19], and WT aSyn amyloid-like fibrils prepared after agitating aSyn (Figure 4, lower panel). By using a sucrose gradient of $10-30 \%$ and an exclusion layer (60\% sucrose) under the gradient, WT aSyn monomers were clearly detected in the low density factions $16-22$. HNE-aSyn and n-aSyn containing oligomers showed a shift of aSyn positive fractions to higher density fractions. Large aSyn aggregates like aSyn fibrils were observed in the first exclusion fractions. We next compared the aSyn positive fractions after SDGC analysis of recombinant WT and aSyn H50 mutants prepared from E. coli. In contrast to WT aSyn, a proportion of aSyn $\mathrm{H} 50$ mutants was detected in the first exclusion fraction with a high sucrose density (60\%), indicating an increased intrinsic aggregation propensity of $\mathrm{H} 50 \mathrm{Q} / \mathrm{R}$ aSyn (Figure 4, upper panel).

To assess the potential existence of soluble, oligomeric species in recombinant aSyn samples, we further analyzed WT and H50 mutant aSyn by using SEC. We did not detect an intrinsic formation of soluble oligomers in untreated WT aSyn and in both H50 aSyn mutants (data not shown). Our previous studies have shown that increased ROS/RNS induce the oxidation/nitration of aSyn. In particular, nitration of aSyn significantly promotes the formation of soluble aSyn oligomers [19]. Thus, we asked whether nitration-mediated aSyn oligomerization is influenced by $\mathrm{H} 50$ mutations. Nitrated aSyn was generated by incubating WT and $\mathrm{H} 50 \mathrm{Q} / \mathrm{R}$ aSyn with tetranitromethane (TNM), an efficient nitrating agent [19]. Nitration triggered the formation of soluble oligomers, which were readily detected in WT aSyn as well as in H50Q/R mutants (Figure 5A). Notably, the H50Q mutation significantly increased the level of TNM-induced oligomeric aSyn compared to WT aSyn (Figure 5B). The H50R mutation also increased nitration-induced oligomerization of aSyn, albeit without reaching statistical significance. This finding demonstrates that H50 mutations exacerbate nitration-mediated

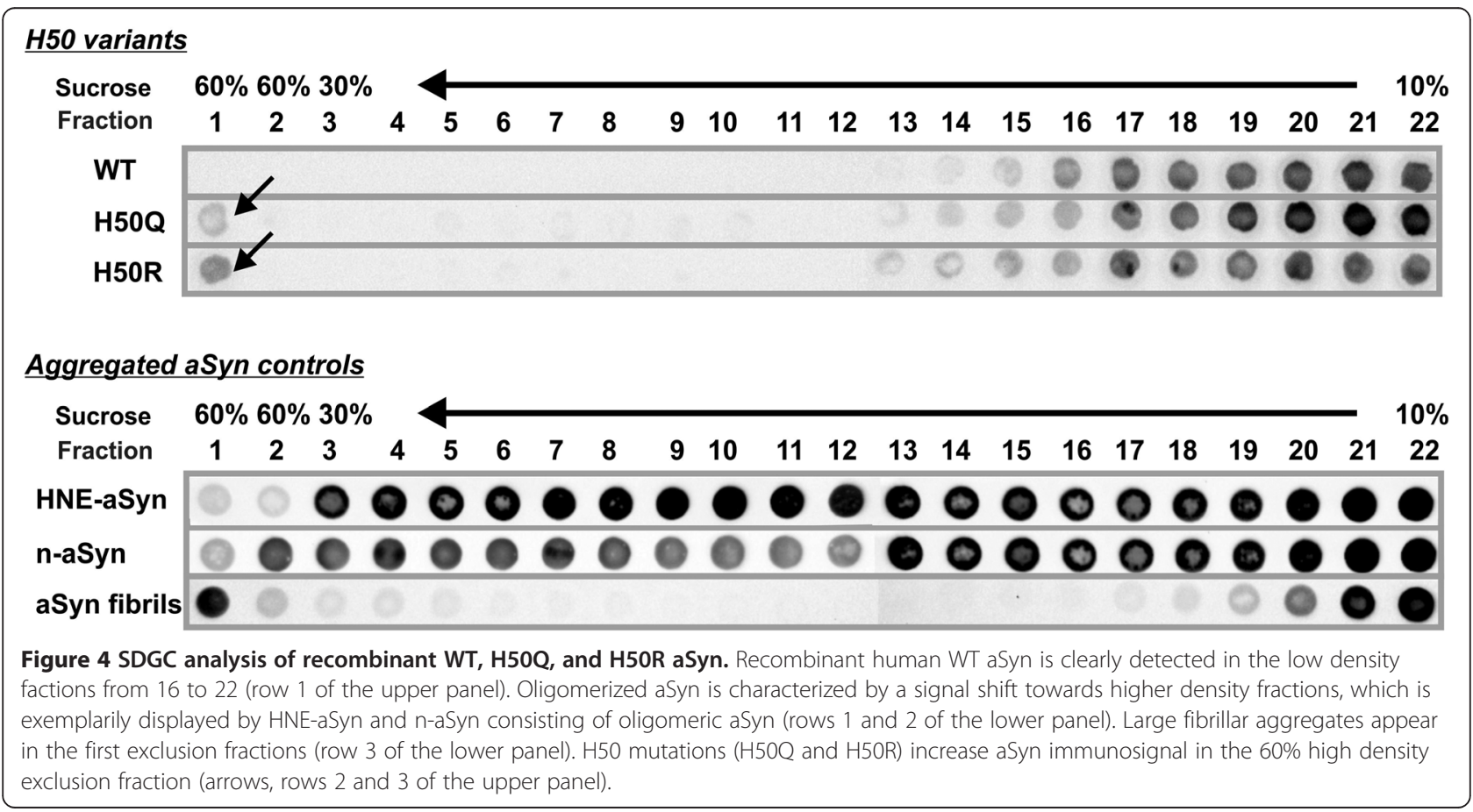




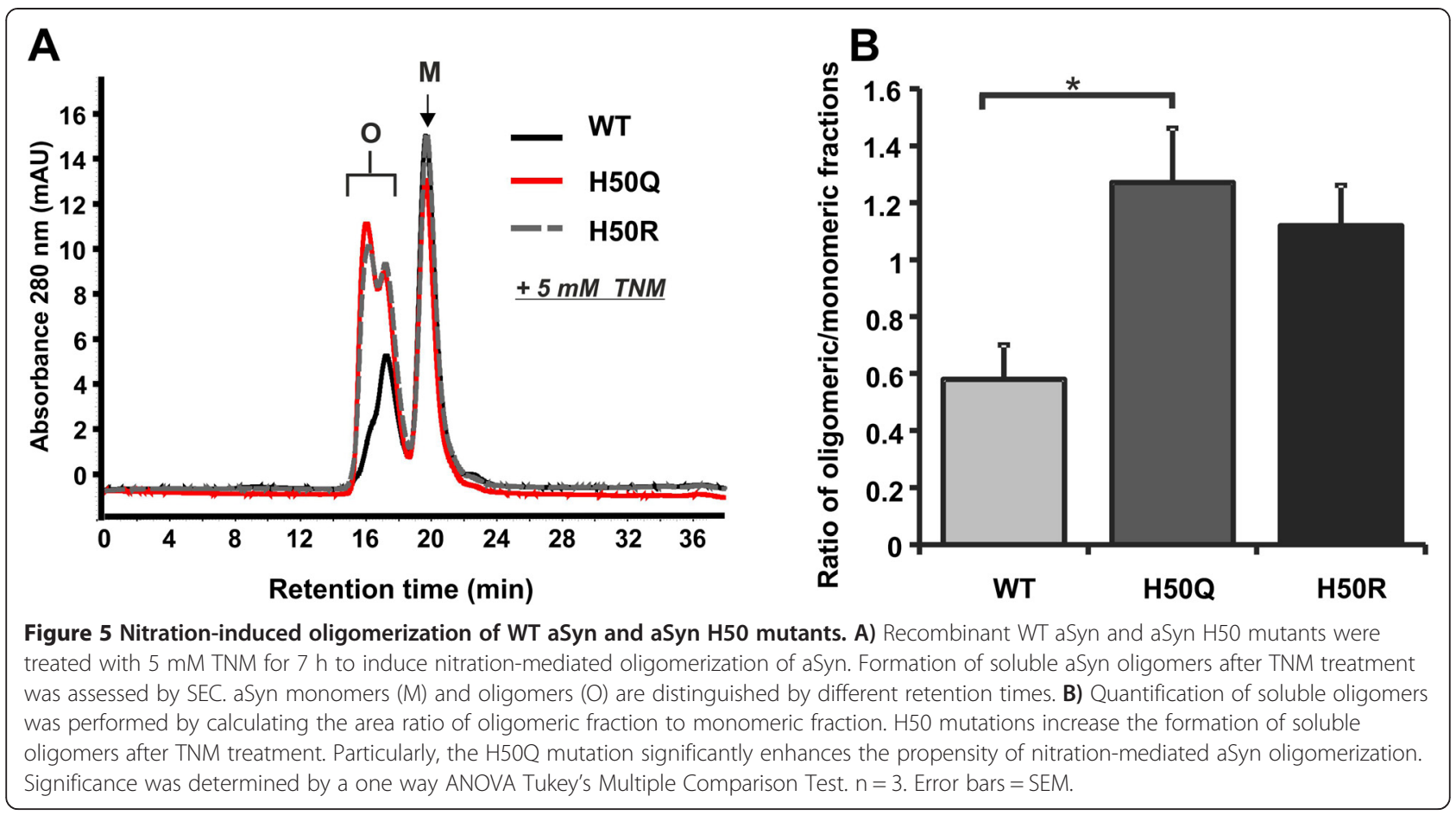

oligomerization, suggesting an increased oligomerization of aSyn H50 mutants under oxidative stress conditions.

In order to study the propensity of H50 mutants to form amyloid fibrils, we generated aSyn fibrils by agitating WT and $\mathrm{H} 50 \mathrm{Q} / \mathrm{R}$ mutant aSyn at $37^{\circ} \mathrm{C}$ for 7 days. Electron microscopy (EM) analysis revealed that WT and H50Q/R
aSyn form fibrillar structures after agitation (Figure 6A). Assessment of amyloid fibrils using Thioflavin T (ThT) assay demonstrated that $\mathrm{H} 50 \mathrm{Q}$ aSyn significantly increases fibrillization compared to WT aSyn both in the absence or presence of $\mathrm{NaCl}$ (Figure 6B). The influence of the H50R mutation on aSyn fibrillization was dependent

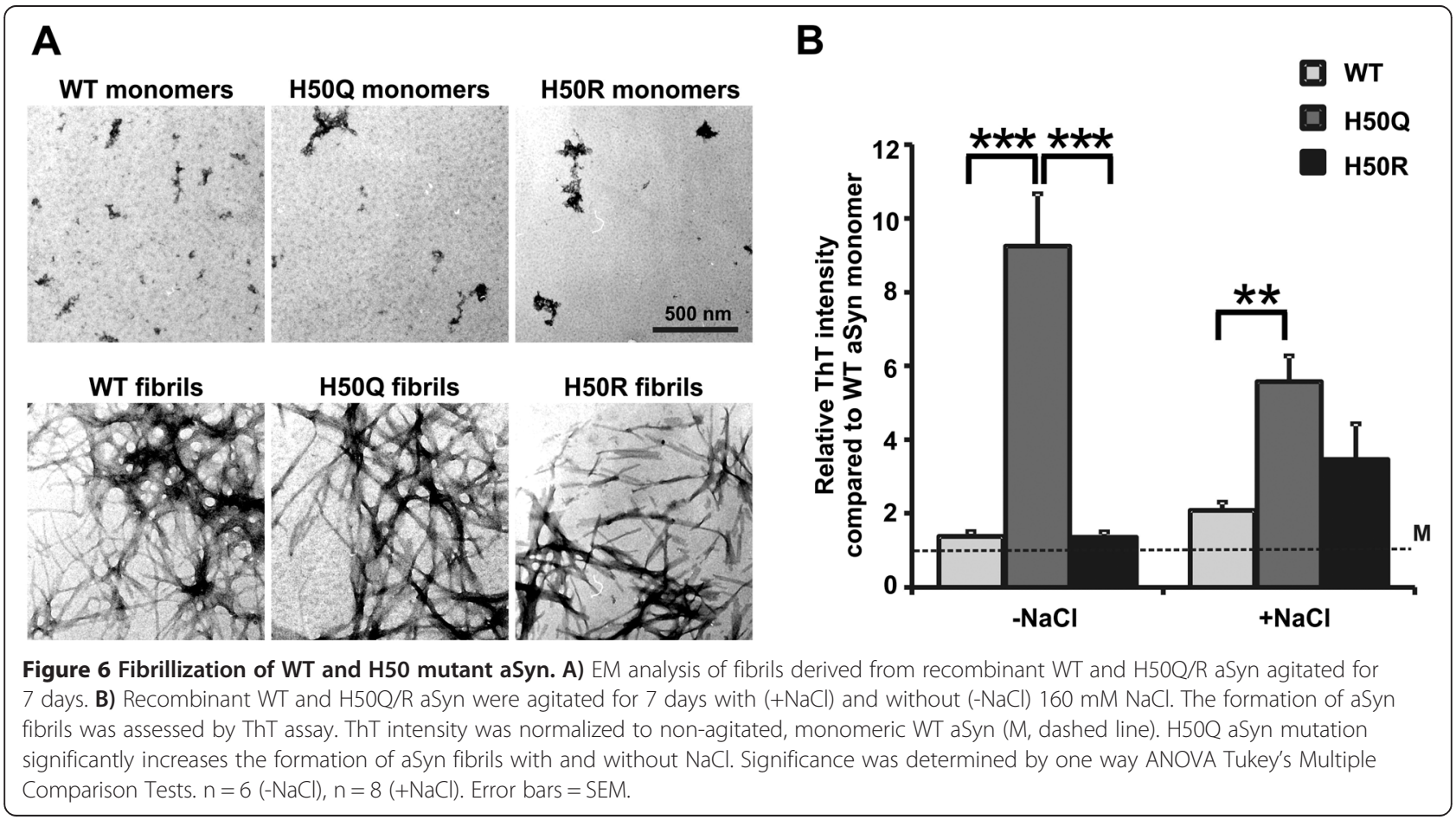


on the salt concentration in the agitation buffer. In the presence of $160 \mathrm{mM} \mathrm{NaCl}$ at a physiological ionic strength the H50R mutant also showed an increase in fibrillization of aSyn, although not reaching statistical significance.

Complementary assessments of aSyn aggregation in vitro revealed that compared to WT aSyn, H50 mutant aSyn is more prone to form 1) high density aggregates (SDGC), 2) soluble oligomers after exposure to a nitrating agent (SEC), and 3) amyloid fibrils after agitation (ThT assay), suggesting a general enhanced aggregation propensity due to $\mathrm{H} 50$ mutations. Thus, we asked if H50 mutations also increase the aggregation propensity of intracellularly expressed aSyn. To this end, we transiently transfected H4 cells with WT and H50Q/R aSyn constructs. The lysates of transfected cells were analyzed by SDGC followed by dot blot analysis. In the lysates of H50Q aSyn transfected cells, we observed a shift of aSyn immunoreactivity from lower to higher density fractions compared to WT aSyn, particularly in fractions $11-17$ (Figure 7). Quantification of aSyn immunointensity from independent analyses revealed a reproducible increase in the aSyn signal in these fractions of higher density for H50Q aSyn samples, confirming an increased oligomerization propensity of cellularly expressed $\mathrm{H} 50 \mathrm{Q}$ mutant aSyn (Figure 7 lower panel). In contrast to the H50Q mutant, the H50R mutant did not show an obvious increase in the aSyn signal in the higher density fractions $11-17$.

\section{Overexpression of $\mathrm{H} 50$ mutant aSyn increases cell damage}

To elucidate whether mutation of H50 increases cytotoxicity of aSyn, we transiently transfected $\mathrm{H} 4$ cells with WT and $\mathrm{H} 50 \mathrm{Q} / \mathrm{R}$ aSyn constructs. Cell damage was assessed by MTS viability assay and ToxiLight toxicity assay. $\mathrm{H} 4$ cells transiently transfected with $\mathrm{H} 50$ aSyn mutants showed a slight reduction in cell viability and an increase in cell death compared to $\mathrm{H} 4$ cells transfected with WT aSyn (Figure 8A). As viability and toxicity assays such as MTS and ToxiLight only determine general damage of transiently transfected cells including overexpressing and non-overexpressing cells, we also used ICC to detect apoptosis specifically in aSyn overexpressing cells (Figure 8B). The number of cells double positive for aSyn and activated (cleaved) Caspase 3 (aCasp3) (aSyn+/aCasp3+) among all aSyn+ cells was determined. Overexpression of $\mathrm{H} 50 \mathrm{Q} / \mathrm{R}$ mutants led to a significant increase in the ratio of cells double positive for aCasp3 and aSyn as compared to the overexpression of WT aSyn, indicating that intracellular overexpression of $\mathrm{H} 50$ mutants promotes apoptosis (Figure 8B).

Since our results showed that overexpression of $\mathrm{H} 50 \mathrm{Q} / \mathrm{R}$ mutant aSyn attenuates cell susceptibility to HNE as compared to WT aSyn (Figure 3, Additional file 1: Figure S2), we asked whether overexpression of H50 mutant aSyn would differently impact the response of cells to another oxidative stressor, e.g. $\mathrm{H}_{2} \mathrm{O}_{2}$. Therefore, we analyzed the influence of overexpression of WT and H50Q/R mutant

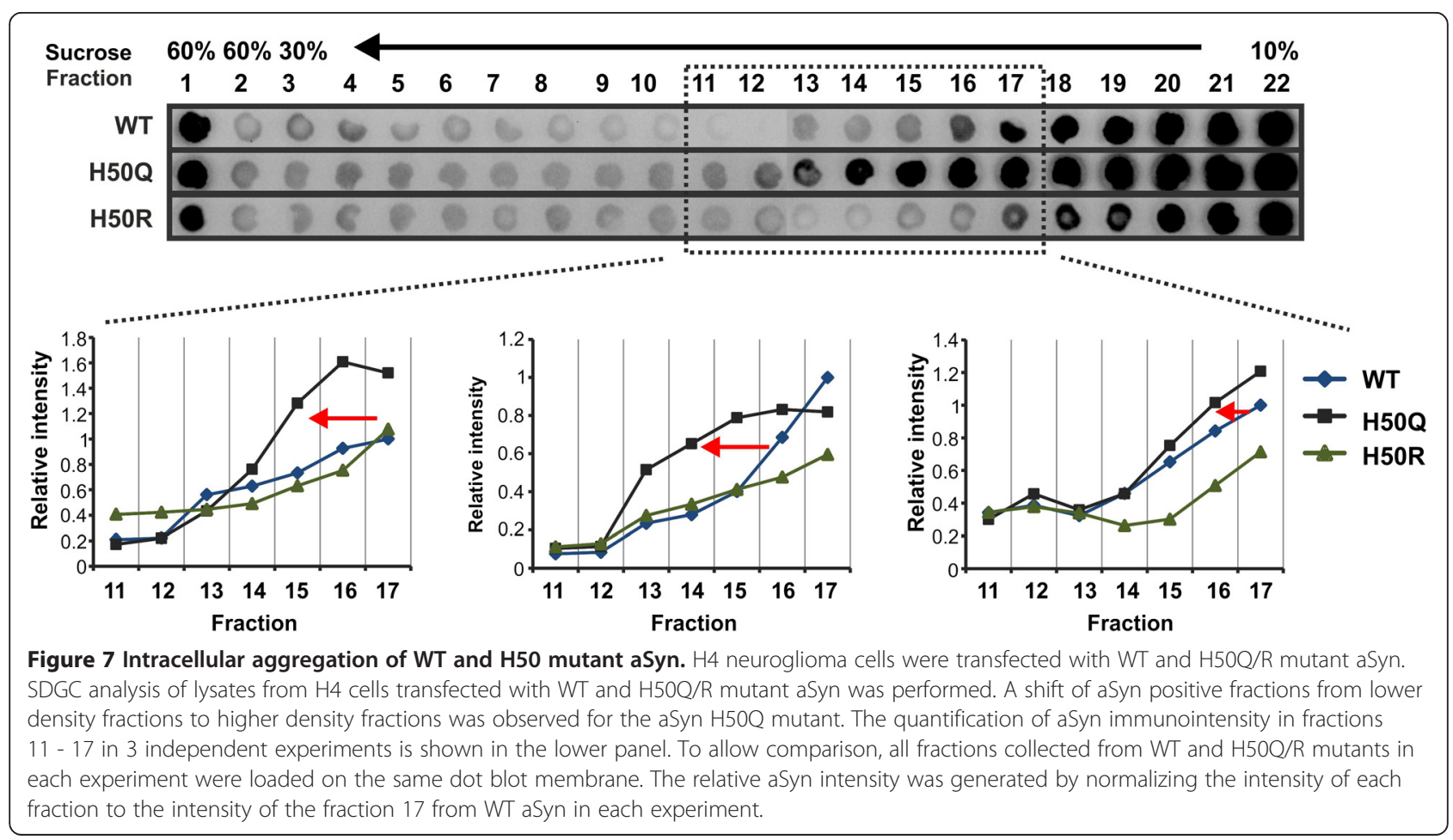



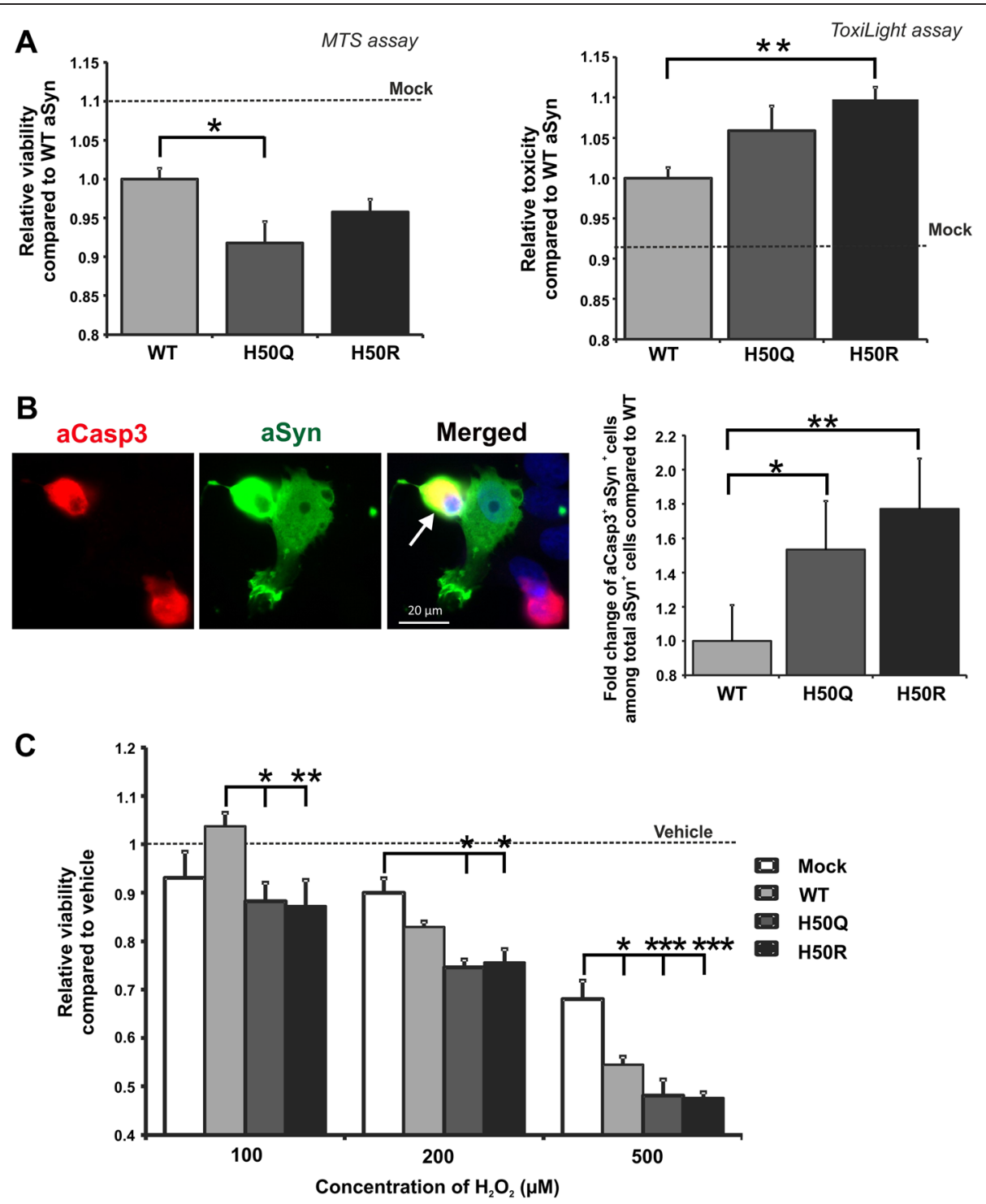

Figure 8 Toxicity of intracellularly overexpressed WT and H50 mutant aSyn. A) H4 neuroglioma cells were transiently transfected with mock, WT, and H50 mutant aSyn, and cell damage was assessed by using MTS cell viability (left) and ToxiLight assays (right). For quantification, measured values were normalized to the mean of WT samples. The background levels of viability and toxicity of mock transfected cells are indicated as dashed lines. Both assays show a slight increase in general cell damage in cells transfected with either H50Q or H50R aSyn. Significance was determined by a one way ANOVA Tukey's Multiple Comparison Test for randomized block experiments. $n=8$ (ToxiLight) and $\mathrm{n}=12$ (MTS assay). Error bars = SEM. B) The induction of apoptosis by overexpressed H50 mutant aSyn was assessed by ICC using antibodies against aSyn (green) and activated Caspase 3 (aCasp3, red). The number of apoptotic, (aCasp3+ aSyn+, arrow) out of total aSyn overexpressing cells (aSyn+) was counted. The proportion of apoptotic cells in H50Q/R aSyn overexpressing cells was significantly higher than in WT aSyn overexpressing cells. For quantification, measured values were normalized to the mean of WT samples. Significance was determined by a one way ANOVA Tukey's Multiple Comparison Test for randomized block experiments. $n=6$. Error bars = SEM. C) H4 cells transfected with mock, WT, and $\mathrm{H} 50$ mutant aSyn were treated with $\mathrm{H}_{2} \mathrm{O}_{2} 24 \mathrm{~h}$ after transfection for another $24 \mathrm{~h}$ followed by MTS viability assay. The viability of treated cells was compared to the viability of the corresponding untreated cells (vehicle, dashed line). $\mathrm{H}_{2} \mathrm{O}_{2}$ treatment induces a more pronounced reduction of the viability of $\mathrm{H} 50 \mathrm{Q} / \mathrm{R}$ overexpressing cells than of WT overexpressing cells. Significance was determined by two way ANOVA Bonferroni Multiple Comparisons. $n \geq 3$. Error bars $=$ SEM.

aSyn on cell viability in the presence of $\mathrm{H}_{2} \mathrm{O}_{2}$, a global inducer of oxidative stress. Application of $\mathrm{H}_{2} \mathrm{O}_{2}$ induced a concentration-dependent decrease in the viability of H4 cells transfected with WT and H50Q/R mutant aSyn (Figure $8 \mathrm{C}$ ). In order to focus on the potential differences in the susceptibility to $\mathrm{H}_{2} \mathrm{O}_{2}$ and thus, to eliminate baseline toxic effects induced by overexpression of $\mathrm{H} 50 \mathrm{mu}$ tant aSyn, the fold reduction in the viability of transfected cells was calculated compared to the corresponding vehicle-treated cells. We observed that the $\mathrm{H}_{2} \mathrm{O}_{2}$ induced 
decline in cell viability was generally more pronounced in $\mathrm{H} 50 \mathrm{Q} / \mathrm{R}$ aSyn than in WT aSyn transfected cells at a $\mathrm{H}_{2} \mathrm{O}_{2}$ concentration range of $100-500 \mu \mathrm{M}$. This effect was statistically significant when using a lower $\mathrm{H}_{2} \mathrm{O}_{2}$ concentration of $100 \mu \mathrm{M}$. This finding suggests that H50 mutations increase the cellular susceptibility to oxidative stress.

\section{Discussion}

In the present study, we used a dual approach to alter the $\mathrm{H} 50$ residue of aSyn, i.e. HNE-PTM and DNA mutation-induced $\mathrm{H} 50$ substitution. We provide an in vitro and cell-based characterization of the impact of these alterations on aSyn aggregation and toxicity, two important features of aSyn pathology, which are crucial for the pathogenesis of PD.

The relevance of HNE modification of aSyn in the pathogenesis of PD is strengthened by several lines of evidence: 1 ) Oxidative stress and lipid peroxidation are strongly associated with neurodegeneration in PD. HNE along with other reactive aldehyde products, such as glyoxal, malondialdehyde, and 4-oxo-2-nonenal (ONE), are important products of lipid peroxidation [18]; 2) The level of HNE-modified proteins is enhanced in affected brain areas, particularly in aSyn containing LBs in PD and other neurodegenerative disorders with LB pathology [24-26]; 3) Several studies have shown that HNE readily modifies aSyn in vitro and in cells exposed to HNE $[19,20,22]$; and 4) HNE modification not only increases aggregation propensity of aSyn in vitro, but HNEmodified aSyn also provokes neuronal damage when applied extracellularly to neurons [19-21]. However, the role of the different HNE target sites of aSyn in these HNE-mediated effects (e.g. aSyn modification, aggregation, and toxicity) has not yet been clarified. Among the possible target sites of HNE, H50 is of particular interest, because a novel aSyn point mutation affecting this residue (H50Q) has recently been reported in monogenic PD patients.

To elucidate the role of H50 in HNE aSyn modification, we analyzed HNE-treated WT and H50Q/R aSyn by using MALDI-TOF MS. The analysis of GluCdigested aSyn variants showed that the $\mathrm{H} 50 \mathrm{Q} / \mathrm{R}$ mutations completely abolish the modification of residue 50 . Importantly, substitution of $\mathrm{H} 50$ remarkably reduced the susceptibility of aSyn to HNE modification, indicating that $\mathrm{H} 50$ is the initial and most reactive residue of aSyn for HNE addition. We have previously shown that HNE-modified aSyn is more prone to form soluble oligomers [19]. Here, we observed significantly reduced formation of oligomers in H50 mutant aSyn compared to WT aSyn by using complementary assays for the analysis of oligomerization, i.e. SEC for the detection of soluble oligomers and SDS-PAGE for the detection of SDS-resistant oligomers. These findings suggest that H50 not only increases the reactivity of aSyn to HNE addition, but also plays a crucial role in HNE-induced aSyn oligomerization.

Based on previous findings showing that HNE modification increases the cytotoxicity of extracellularly applied aSyn [19-21] and that extracellular application of HNE causes HNE modification of intracellularly expressed aSyn [22], we hypothesized that overexpression of aSyn increases the susceptibility of cells to HNE due to the formation of intracellular H50-dependent HNE-aSyn modification. To verify this hypothesis, we overexpressed WT and H50Q/R aSyn in H4 cells and compared cell susceptibility to HNE by using two complementary methods, i.e. MTS viability assay and flow cytometric analysis. Our results indicate that the substitution of $\mathrm{H} 50$, the most reactive residue for $\mathrm{HNE}$ modification, reduces the susceptibility of aSyn overexpressing cells to HNE-induced toxicity. Thus, our experiments not only provide evidence that $\mathrm{H} 50$ plays a crucial role for HNE-mediated modification and oligomerization of aSyn, but also suggest its implication in HNE-induced cytotoxicity, probably due to HNE modification of intracellular aSyn at the $\mathrm{H} 50$ residue.

The identification of the novel PD-associated aSyn H50Q mutation provides evidence for the relevance of aSyn $\mathrm{H} 50$ not only in sporadic but also in monogenic PD. Thus, we asked whether the H50 mutations intrinsically potentiate aSyn pathology, despite reducing HNEmediated aSyn aggregation and toxicity, thereby supporting the pathogenicity of the novel reported $\mathrm{H} 50 \mathrm{Q}$ mutation. We analyzed the effects of H50 mutations on aSyn aggregation both in vitro using recombinant human aSyn, and in aSyn overexpressing cells. Analysis of the formation of amyloid fibrils in agitated aSyn samples using ThT assay demonstrated that H50Q mutation significantly increases the fibrillization of aSyn compared to WT aSyn, which is consistent with recent in vitro studies [27-29]. In contrast to a recent study [30], H50R did not significantly reduce fibrillization compared to WT aSyn in our experiments, likely due to different conditions for the fibrillization experiments (e.g. starting concentration of monomeric aSyn and buffer composition). In our study, the salt concentration in the aggregation buffer influenced the impact of the H50R aSyn mutation on fibrillization. Under physiological salt concentrations, H50R aSyn also showed higher fibrillization levels than WT aSyn, although less pronounced than H50Q aSyn. Consistently, we detected aSyn $\mathrm{H} 50 \mathrm{Q} / \mathrm{R}$ in the first high density fractions of SDGC, analogous to amyloid fibrils. These findings indicate that $\mathrm{H} 50 \mathrm{Q} / \mathrm{R}$ mutations intrinsically increase the propensity of aSyn to form larger aggregates. Yet, accumulating evidence suggests that small aggregation intermediates, especially oligomeric aSyn species, may be more toxic than large amyloid fibrils [31-33]. Therefore, in addition to fibrillization, we 
investigated the formation of oligomers. We did not detect soluble oligomers in unmodified recombinant WT aSyn and $\mathrm{H} 50 \mathrm{Q} / \mathrm{R}$ mutants by applying SEC. However, we were able to show that $\mathrm{H} 50 \mathrm{Q} / \mathrm{R}$ mutations, particularly the $\mathrm{H} 50 \mathrm{Q}$ mutation, increased the formation of soluble aSyn oligomers in the presence of a nitrating agent, indicating that H50 mutant aSyn is more prone to form soluble oligomers under oxidative stress, characterized by accumulating ROS/RNS. Recently, it was shown that the H50Q mutation leads to a chemical shift within the C-terminal region (residue 135-140) of aSyn [30], affecting tyrosine 136, one target site for nitration [19]. Thus, H50 mutation may directly influence the accessibility or reactivity of this tyrosine residue to ROS/ RNS, and consequently increase oligomerization propensity of aSyn in response to oxidative stress.

To validate our in vitro observation concerning the influence of $\mathrm{H} 50$ mutations on aSyn aggregation, we analyzed the impact of these mutations on intracellularly overexpressed aSyn in H4 cells. In a very recent study, in which the oligomerization of various aSyn mutants/variants was examined in HEK cells by using Bimolecular Fluorescence Complementation (BiFC) assay, no significant differences between WT and H50Q aSyn have been detected [34]. Interestingly, we observed an increase in H50Q aSyn immunosignal in higher density fractions of SDGC, suggesting an enhanced formation of oligomeric aSyn species in $\mathrm{H} 4$ cells. Taken together, our in vitro and cell-based experiments suggest that H50Q mutation intrinsically increases aggregation propensity of aSyn when compared to WT. Yet, while H50Q aSyn tends to form larger fibrillar aggregates in vitro as revealed by ThT and SDGC analysis, intracellularly overexpressed H50Q aSyn is more prone to the formation of oligomeric species as indicated by SDGC analysis. This discrepancy may be attributed to the presence of cellular factors that impact the aggregation kinetics of aSyn in cells, which is in accordance to our in vitro finding that additional treatment of aSyn with a nitrating agent increases the propensity of H50 mutant aSyn to form oligomers. Moreover, protein degradation pathways may also influence aSyn aggregation. In particular, we have recently shown that oligomeric and higher aggregated aSyn species are differently processed by autophagy as a major protein degradation pathway [33]. Therefore, intracelluar oligomerization of H50 mutant aSyn may be affected by autophagy associated pathways, which would be important to address in future studies.

To study the toxic effect of intracellularly expressed aSyn H50 mutants, we overexpressed WT and H50Q/R aSyn mutants in $\mathrm{H} 4$ cells by transient transfection. We generally observed a slight increase in cell damage due to $\mathrm{H} 50 \mathrm{Q} / \mathrm{R}$ mutations as measured either by ToxiLight toxicity or by MTS viability assay. In a more specific assessment of the direct effect of overexpressed aSyn on toxicity, we focused on aSyn overexpressing cells and analyzed the proportion of apoptotic cells among aSyn+ cells (aCasp3+/aSyn+). This specific cell death assay and the cell experimental conditions used in this study allowed us to detect a more pronounced toxic effect evoked by intracellularly overexpressed $\mathrm{H} 50 \mathrm{Q} / \mathrm{R}$ mutants, which was not revealed in other recent studies on pathological cellular effects of H50Q mutation $[27,28]$. We furthermore analyzed the influence of $\mathrm{H} 50 \mathrm{Q} / \mathrm{R}$ mutation on the $\mathrm{H}_{2} \mathrm{O}_{2}$-induced reduction of cellular viability. Our results showed that overexpression of $\mathrm{H} 50 \mathrm{Q} / \mathrm{R} \mathrm{mu-}$ tant aSyn increases cellular susceptibility to $\mathrm{H}_{2} \mathrm{O}_{2}$ compared to WT aSyn. This finding is in agreement with a previous study observing this effect for $\mathrm{H} 50 \mathrm{Q}$ aSyn in another cell model [27]. Using a $\mathrm{H}_{2} \mathrm{O}_{2}$ concentration range between $100-500 \mu \mathrm{M}$, we noticed that the increase in cellular susceptibility to oxidative stress due to $\mathrm{H} 50 \mathrm{Q} / \mathrm{R}$ mutation is especially marked at a lower $\mathrm{H}_{2} \mathrm{O}_{2}$ concentration (i.e. $100 \mu \mathrm{M}$ ) when compared to WT aSyn. The difference in cellular susceptibility between aSyn H50 mutants and WT aSyn declines with increasing $\mathrm{H}_{2} \mathrm{O}_{2}$ concentrations (i.e. $200-500 \mu \mathrm{M}$ ). This might be explained by the occurrence of global damage under strong oxidative stress conditions. Thus, our results suggest that the aSyn H50 mutation particularly increases cellular susceptibility at comparatively low levels of oxidative stress. Taking into account that oxidative stress conditions potentiate the formation of aSyn oligomers in H50 mutant aSyn (particularly H50Q aSyn) in vitro, our study indicates that the increased toxicity of $\mathrm{H} 50 \mathrm{Q} / \mathrm{R}$ mutant aSyn under oxidative stress might be associated with the formation of toxic oligomeric aSyn species.

Interestingly, $\mathrm{H} 50 \mathrm{Q} / \mathrm{R}$ mutant aSyn differentially influences cellular response to $\mathrm{HNE}$ and $\mathrm{H}_{2} \mathrm{O}_{2}$. Both stressors trigger cell death in a dose-dependent manner. However, while $\mathrm{H} 50 \mathrm{Q} / \mathrm{R}$ overexpressing cells appear to be more resistant to HNE than the WT aSyn overexpressing cells, they are more vulnerable to $\mathrm{H}_{2} \mathrm{O}_{2}$. These findings emphasize the role of $\mathrm{H} 50$ as an important HNE modification site in HNE-mediated toxicity, with particular relevance for sporadic PD. Additionally, the increased susceptibility to $\mathrm{H}_{2} \mathrm{O}_{2}$ due to $\mathrm{H} 50$ mutations suggests that the mechanistic role of the $\mathrm{H} 50 \mathrm{Q}$ mutation in monogenic PD may rely on an altered neuronal response to general oxidative stress. To clarify whether H50 mutation generally increase cellular susceptibility to insults or whether this effect is specific to oxidative stress, further extensive studies are needed analyzing the impact of $\mathrm{H} 50 \mathrm{Q}$ mutation under more specified oxidative stress and non-oxidative stress related conditions.

In this study, we substituted $\mathrm{H} 50$ by two amino acid residues with different chemical characteristics, i.e. the 
uncharged and polar glutamine and the basic arginine (like histidine). Both mutations impact aggregation behavior and toxicity of aSyn, although to a different extent. Thus, our results suggest that the effects of the PD-related H50Q mutation are attributed to the loss of a functional $\mathrm{H} 50$ residue rather than to a specific gain of function induced by the glutamine. Nevertheless, the H50R mutation appeared to be more toxic than the H50Q mutation, although H50R aSyn was less prone to aggregation than $\mathrm{H} 50 \mathrm{Q}$ aSyn in vitro, and increased intracellular aggregation of H50R aSyn was not detectable by using SDGC. These interesting findings suggest that besides aggregation, other mechanisms affecting the physiological or pathological function of aSyn have to be considered when investigating H50-mutation dependent toxicity.

\section{Conclusion}

Our data demonstrate that aSyn $\mathrm{H} 50$ alterations, either by oxidative stress-related HNE modification or by gene mutation, increase aggregation propensity and cytotoxicity of aSyn (Figure 9). Moreover, we reveal that oxidative stress not only triggers aSyn pathology by directly modifying $\mathrm{H} 50$ of WT aSyn via HNE addition, but also potentiates the pathological effects of the H50Q mutation. Although H50Q mutation diminishes the HNE-mediated effects on aSyn, it intrinsically increases aSyn aggregation and toxicity, and exacerbates the cellular susceptibility to oxidative stress, especially to ROS/RNS other than the lipid peroxidation product HNE. Thus, we provide novel insights into the mechanisms underlying oxidative stress-associated pathogenesis of sporadic and H50Qassociated monogenic PD.

\section{Methods}

\section{Site-directed mutagenesis}

The QuikChange ${ }^{\oplus}$ Site-directed Mutagenesis Kit (Agilent Technologies, Waldbronn, Germany) was applied to generate both aSyn $\mathrm{H} 50 \mathrm{Q} / \mathrm{R}$ mutations in the coding sequence of WT aSyn in human aSyn pcDNA3.1 and pT7-7 constructs according to the manufacturer's protocol. The mutation-covering, complementary primer sequences 5' G GAG GGA GTG GTG CAG GGT GTG GCA ACA G 3' and 5' C TGT TGC CAC ACC CTG CAC CAC TCC CTC C 3', as well as 5' G GAG GGA GTG GTG CGT GGT GTG GCA ACA G 3' and 5' C TGT TGC CAC ACC ACG CAC CAC TCC CTC C 3' were used to generate aSyn H50Q and H50R mutations, respectively.

\section{Preparation of recombinant human aSyn and induction of PTMs}

Constructs containing WT aSyn and aSyn H50 mutants were transformed in E. coli BL21 (DE3) pLys competent cells (Novagen, San Diego, CA, USA). The preparation and purification of recombinant WT and H50Q/R mutant aSyn were performed as previously described [19]. To induce nitration, recombinant aSyn of $70 \mu \mathrm{M}$ was incubated with $5 \mathrm{mM}$ tetranitromethane (TNM) in $30 \mathrm{mM}$ Tris/HCl pH 7.4 at room temperature (RT) for 7 h. HNEmodified aSyn was generated by incubating aSyn with $\mathrm{HNE}$ concentrations ranging from $50 \mu \mathrm{M}$ to $3000 \mu \mathrm{M}$ in $30 \mathrm{mM}$ Tris $/ \mathrm{HCl} \mathrm{pH} 7.4$ at $37^{\circ} \mathrm{C}$ for $24 \mathrm{~h}$.

\section{Mass spectrometry}

Detection of HNE-modified aSyn was performed by matrix-assisted laser-desorption-ionization time-of-flight mass spectrometry (MALDI-TOF MS) as previously described [19]. Briefly, for the digestion with GluC

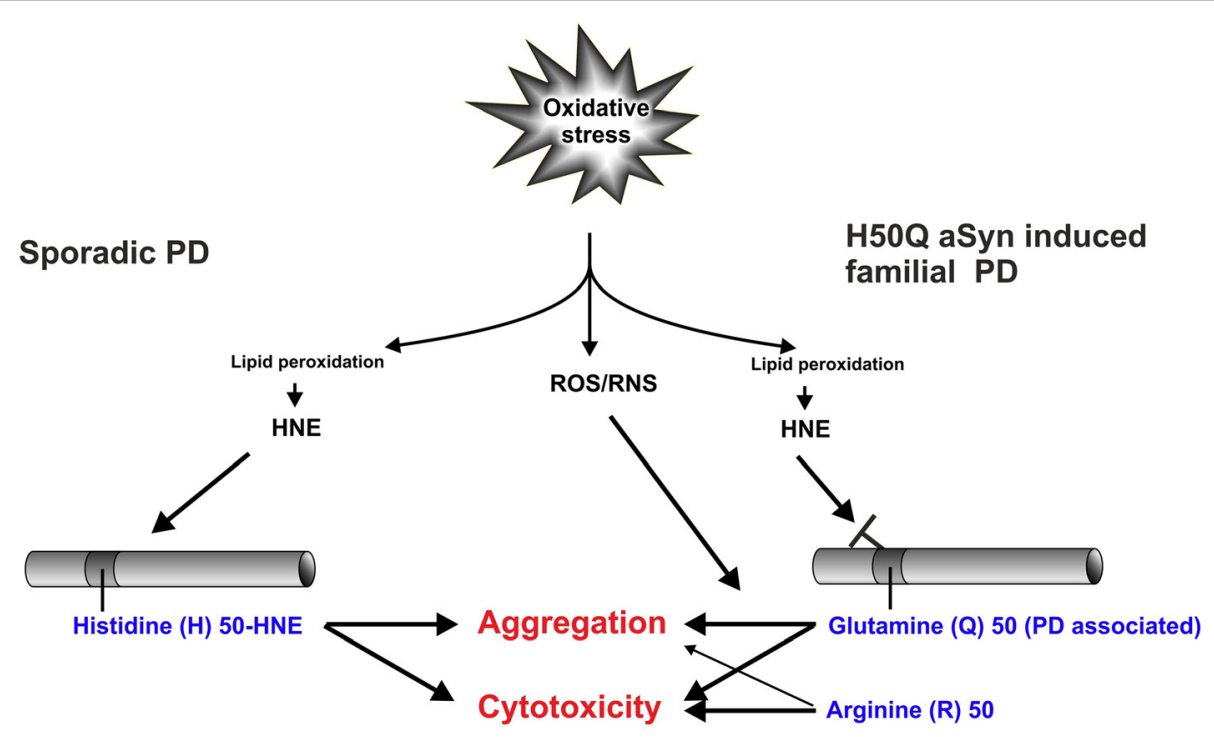

Figure 9 Summary of the impact of aSyn $\mathrm{H} 50$ modification and mutation on aSyn pathology. 
endoproteinase (Roche Diagnostics GmbH, Penzberg, Germany) 3-5 $\mu$ g recombinant aSyn and 500 ng GluC was dissolved in $50 \mathrm{mM} \mathrm{NH}_{4} \mathrm{HCO}_{3}$ and incubated overnight at RT. Full-length aSyn or GluC-digested protein samples were mixed with $0.1 \%$ trifluoroacetic acid (TFA) $(\mathrm{v} / \mathrm{v})$ and MALDI matrix 2,5-dihydroxyacetophenone, spotted on a stainless steel target, and measured by a Bruker Autoflex (Bruker Daltonik, Bremen, Germany). Positive ions were analyzed in reflector mode after acceleration by $20 \mathrm{kV}$. External calibration was performed using a peptide calibration standard (Bruker Daltonik). Each displayed mass spectrum was produced by five individual spectra, which were generated by 50 shots/ individual spectrum recorded from several positions on a spot. Spectra were analyzed using Flex Analysis software (Bruker Daltonik). Mass of aSyn ions or GluCdigested aSyn fragments are given in the mass of singly charged $[\mathrm{M}+\mathrm{H}]^{+}$ions.

\section{Size exclusion chromatography}

Size exclusion chromatography (SEC) was applied to assess soluble oligomeric aSyn. Prior to SEC, samples were centrifuged at $100000 \mathrm{~g}$ for $60 \mathrm{~min}$. aSyn species were separated on a SuperdexTM 75 10/300 column (GE Healthcare, Freiburg, Germany) using $30 \mathrm{mM}$ Tris/HCl, $0.2 \mathrm{M} \mathrm{NaCl}, \mathrm{pH} 7.4$ as an eluent at a flow rate of $0.5 \mathrm{ml} /$ min and monitoring the UV absorbance at $280 \mathrm{~nm}$. To ensure reproducibility between SEC runs, Gel Filtration Standard (Bio-Rad Laboratories, Munich, Germany) was used prior to each set of analysis.

\section{In vitro fibrillization of aSyn and Thioflavin $\mathrm{T}$ assay}

WT and $\mathrm{H} 50 \mathrm{Q} / \mathrm{R}$ mutant aSyn of $70 \mu \mathrm{M}$ in $30 \mathrm{mM}$ Tris/ $\mathrm{HCl} \mathrm{pH} \mathrm{7.4} \mathrm{(100} \mu$ l per sample) were incubated in a 96-well plate and agitated at $200 \mathrm{rpm}$ on a Controlled Environmental Incubator Shaker (New Brunswick Scientific INC, New Brunswick, NJ, USA) at $37^{\circ} \mathrm{C}$ for $7 \mathrm{~d}$ in the presence of glass beads with a diameter of $2 \mathrm{~mm}$. For the detection of aSyn fibrils, $6 \mu \mathrm{g}$ of agitated aSyn was diluted in $30 \mathrm{mM}$ Tris/ $\mathrm{HCl} \mathrm{pH} 7.4$ containing $20 \mu \mathrm{M}$ ThT (Sigma-Aldrich, Taufkirchen, Germany) and incubated for $10 \mathrm{~min}$ at $300 \mathrm{rpm}$ and RT in the dark. The fluorescence intensities were measured in a Varian Cary Eclipse fluorescence spectrophotometer (Agilent Technologies, Waldbronn, Germany) with an excitation at $445 \mathrm{~nm}$ and an emission at $480 \mathrm{~nm}$. For quantification, values were normalized to the fluorescence intensity of non-agitated WT aSyn.

\section{Electron microscopy}

Electron microscopy (EM) was performed to analyze fibrillization of WT and H50 mutant aSyn. For negative staining, $10 \mu \mathrm{l}$ of each agitated aSyn sample at a concentration of $70 \mu \mathrm{M}$ were adsorbed onto glow-discharged carbon-coated Formvar grids (Electron Microscopy Sciences, Hatfield, PA, USA), incubated for $20 \mathrm{~min}$, washed with distilled water, and then stained with a filtered $3 \%$ aqueous uranyl acetate solution for $5 \mathrm{~min}$. After another washing step, grids were dried and analyzed with a transmission electron microscope (LEO 906E; Carl Zeiss, Oberkochen, Germany).

\section{Cell culture, calcium-phosphate transfection, and cell treatment}

H4 neuroglioma cells of human origin (ATCC, HTB-148) were maintained in Opti-MEM + GlutaMAX (51985-042, Invitrogen, Darmstadt, Germany) supplemented with $10 \%$ fetal calf serum (FCS; 10270-106, Invitrogen, Darmstadt, Germany) at $37^{\circ} \mathrm{C} .24 \mathrm{~h}$ prior transfection cells were plated in 24-well plates $\left(3.5 \times 10^{4}\right.$ cells/well $)$ or in 6-well plates $\left(2 \times 10^{5}\right.$ cells/well $)$ and cultured in $0.5 \mathrm{ml}$ or $2 \mathrm{ml}$ medium, respectively. Calcium-phosphate transfection was performed as previously described [35]. Equimolar ratios of pcDNA3.1 plasmids encoding human WT, H50Q, and H50R aSyn were transfected under a cytomegalovirus promoter. Mock transfection without adding plasmid DNA served as control. The transfection efficiency was controlled by using flow cytometry. For the treatment experiments, $\mathrm{H} 4$ cells were exposed to different concentrations of HNE $(50-3000 \mu \mathrm{M}$, Cayman Chemical Company, Ann Arbor, MI, USA) or $\mathrm{H}_{2} \mathrm{O}_{2}(100-500 \mu \mathrm{M}$, Merck, Darmstadt, Germany) 24 h after transfection for $12 \mathrm{~h}$ ( $\mathrm{HNE})$ or $24 \mathrm{~h}\left(\mathrm{H}_{2} \mathrm{O}_{2}\right)$, respectively. The stock solution of $64 \mathrm{mM}$ HNE was prepared in ethanol. The stock solutions of $\mathrm{HNE}$ and $\mathrm{H}_{2} \mathrm{O}_{2}$ were freshly diluted in culture medium prior to treatment. Vehicle controls (ethanol or culture medium) were prepared in accordance to the highest $\mathrm{HNE}$ and $\mathrm{H}_{2} \mathrm{O}_{2}$ concentration used for the treatments.

\section{Detection of aSyn transfection efficiency and expression levels via flow cytometry}

$\mathrm{H} 4$ cells were seeded in 24 -well plates $\left(3.5 \times 10^{4}\right.$ cells per well), transfected with pcDNA3.1 expression vectors encoding WT and $\mathrm{H} 50 \mathrm{Q} / \mathrm{R}$ mutant aSyn, and harvested $24 \mathrm{~h}$ after transfection. Cells were washed with PBS and fixed by using a 1:4 dilution of Fixation/Permeabilization Concentrate (eBioscience, Frankfurt, Germany) in Fixation/ Permeabilization Diluent (eBioscience). Cells were washed with Permeabilization Buffer (eBioscience) and blocked for 15 min by using $10 \mu \mathrm{l}$ of FcR Blocking Reagent (human, Miltenyi Biotech, Bergisch Gladbach, Germany). Detection of aSyn was performed by applying a rat anti-human aSyn primary antibody (1 h, 1:400, Enzo Life Sciences, ALX-804258-L001, Lörrach, Germany) and an Alexa488-labeled donkey anti-rat secondary antibody (1 h, 1:800, A21208, Invitrogen, Darmstadt, Germany) diluted in Permeabilization Buffer. 20000 cells were detected to analyze aSyn 
transfection efficiency and the mean intensity of the aSyn signal via flow cytometry with a CyFlowR Space (Partec, Münster, Germany) and the FloMax 2.81 analysis and quantification software. The forward and sideward scatter signal was used to determine the population of single cells used for measurements. The cut-off fluorescence intensity for defining aSyn expressing cells was set by measuring mock transfected cells treated with the same staining protocol.

\section{Assessment of toxicity and cell viability}

Toxicity of aSyn transfected H4 cells was analyzed in 24-well plates by ToxiLight enzyme activity assay for membrane integrity (Lonza, Basel, Switzerland), and MTS viability assays (Promega, Mannheim, Germany) according to the manufacturer's protocol, $36 \mathrm{~h}$ and $48 \mathrm{~h}$ after transfection, respectively.

In order to measure apoptotic cell death, activated (cleaved) Caspase 3 positive cells were assessed by ICC. For this, $\mathrm{H} 4$ cells were plated on $13 \mathrm{~mm}$ glass coverslips prior to transfection. $36 \mathrm{~h}$ after transfection, cells were fixed with $4 \%$ paraformaldehyde for $15 \mathrm{~min}$. Cells were washed with Tris buffered saline (TBS, $\mathrm{pH}$ 7.4) and blocked with fish skin gelatin buffer (FSGB) containing $50 \mathrm{mM}$ Tris $/ \mathrm{HCl}, \mathrm{pH} 7.4,1 \%$ BSA, $0.2 \%$ fish skin gelatin, and $0.1 \%$ Triton-X 100 for $1 \mathrm{~h}$ at RT. Cells were incubated with primary rat anti-human aSyn (1:250, Enzo Life Sciences, ALX-804-258-L001, Lörrach, Germany) and rabbit anti-aCasp3 (1:500, 9661, Cell Signaling Technology, Danvers, MA, USA) antibodies overnight at $4^{\circ} \mathrm{C}$. After washing, Alexa 488-labeled donkey anti-rat secondary antibody (1:1000, A21208, Invitrogen, Darmstadt, Germany) and Alexa 568labeled donkey anti-rabbit secondary antibody (1:1000, A10042, Invitrogen) were applied for $1 \mathrm{~h}$ at RT. Nuclei were counterstained with 4'6' -diamidino2-phenylindol (DAPI, 1:10000, D8417, Sigma-Aldrich, Steinheim, Germany) for 15 min. After washing, coverslips were mounted by using Prolong Antifade reagent (P36930, Invitrogen). The ratio of cells positive for aCasp3 (aCasp3+) among aSyn positive (aSyn + ) cells was assessed in accordance to a systematic, random counting procedure [19]. For image acquisition, Axio Imager M2 microscope combined with an AxioCam MRm camera (Carl Zeiss AG, Jena, Germany) with the same settings and exposure times within each independent experiment was used. Six images of each coverslip were randomly selected at $20 \times$ magnification to enable the analysis of at least 200 aSyn positive cells per independent experiment. aSyn + and aCasp3+ cells were scored based on the presence of immunostaining compared to the background staining of the corresponding controls.

\section{SDS-PAGE and Western blot}

For Western blot (WB) of recombinant aSyn, $0.5 \mu \mathrm{g}$ aSyn was mixed with one volume of SDS sample buffer (0.125 M Tris/HCl pH 6.8, 4\% SDS, 20\% glycerol), separated on 15\% SDS-PAGE, and blotted onto nitrocellulose membranes (Millipore, Darmstadt, Germany). The blots were probed with a mouse anti-aSyn primary antibody (Syn-1, 1:2000, BD Transduction Laboratories, San Diego, CA, USA) or a rabbit anti-aSyn antibody (SNCA antibody, 1:2000, Proteintech Europe, Manchester, UK). While Syn-1 was generated using aSyn fragment amino acids $15-123$ as antigen, SNCA antibody was raised against full-length aSyn. The nitrocellulose membranes were subsequently probed with secondary goatanti-mouse antibody or goat anti-rabbit antibody coupled to horseradish peroxidase (1:10000 Dianova, Hamburg, Germany). For the detection of proteins, membranes were incubated with the SuperSignal West Pico or Femto Sensitivity Substrate ${ }^{\mathrm{Tx}}$ (Thermo Scientific Rockford, lL, USA). Immunoblots were visualized by VersaDoc gel imaging system (BioRad, Munich, Germany).

\section{Sucrose density gradient centrifugation and dot blot}

Sucrose density gradient centrifugation (SDGC) was performed as previously described [36] with minor modifications. Briefly, a continuous $10-30 \%$ sucrose gradient $(30 \mathrm{ml})$ in $25 \mathrm{mM}$ Tris- $\mathrm{HCl}, \mathrm{pH} 7.4,0.2 \mathrm{M} \mathrm{NaCl}$ was prepared on top of the $60 \%$ sucrose cushion $(4 \mathrm{ml})$. For analyzing recombinant aSyn samples, $30 \mu \mathrm{g}$ of aSyn was loaded on top of the sucrose gradient. For analyzing aggregation of cellular expressed aSyn, $\mathrm{H} 4$ cells were seeded in a 6 well plate $\left(2 \times 10^{5}\right.$ cells per well $)$ for transfection. Cells from 3 wells were collected $36 \mathrm{~h}$ after transfection via scraping in ice-cold PBS containing protease/phosphatase inhibitors and pooled for SDGC analysis. Subsequently, cell pellets were homogenized in $50 \mathrm{mM}$ Tris/ $\mathrm{HCl} \mathrm{pH} 7.4$ buffer containing $150 \mathrm{mM} \mathrm{NaCl}, 2 \mathrm{mM}$ EDTA, 1\% (v/v) NP$40,0.1 \%(\mathrm{w} / \mathrm{v})$ SDS, and complete mini protease inhibitors cocktail (Roche Diagnostics $\mathrm{GmbH}$ ) in a Potter dounce homogenizer at $4^{\circ} \mathrm{C}$. The lysates were loaded on top of the sucrose gradient. After centrifugation in a Beckman L-70 Ultracentrifuge with a SW-28 rotor (Beckman Coulter, Brea, CA, USA) at $26000 \mathrm{rpm}$ for $18 \mathrm{~h}$ at $4^{\circ} \mathrm{C}, 22$ sucrose fractions with $1.5 \mathrm{ml}$ each were collected. $400 \mu \mathrm{l}$ of each fraction were mixed with $100 \mu \mathrm{l}$ methanol and spotted on a nitrocellulose membrane. After blocking the nitrocellulose membranes, aSyn was visualized using a mouse antiaSyn primary antibody (Syn-1, 1:2000, BD Transduction Laboratories), and a HRP-conjugated goat anti-mouse secondary antibody (1:10000, Dianova). For the detection of aSyn in SDGC fractions, membranes were incubated with the SuperSignal West Pico or Femto Sensitivity Substrate ${ }^{\text {ma }}$ (Thermo Scientific). 


\section{Statistical analysis}

Statistical analyses were performed using GraphPad Prism (GraphPad Software, San Diego, CA, USA). All numeric results are reported as mean + standard error of the mean (SEM) and represent data from a minimum of three independent experiments unless otherwise stated. Significant differences are depicted in the figures by graphical representation. $\mathrm{p}<0.05$ was considered as significant $=$ $* \mathrm{p}<0.01=* * \mathrm{p}<0.001=* * *$.

\section{Additional file}

Additional file 1: Figure S1. Determination of transfection efficiency by flow cytometry. Figure S2. Analysis of the susceptibility of WT and H50 mutant aSyn overexpressing cells to HNE by flow cytometry.

\begin{abstract}
Abbreviations
aCasp3: Activated (cleaved) Caspase 3; aSyn: Alpha synuclein; BCA: Bicinchonic acid; BSA: Bovine serum albumin; DAPI: 4',6'-Diamidino-2-phenylindol; EDTA: Ethylenediaminetetraacetic acid; EM: Electron microscopy; FCS: Fetal calf serum; FSGB: Fish skin gelatin buffer; HNE: 4-hydroxy-2-nonenal; $\mathrm{H}_{2} \mathrm{O}_{2}$ : Hydrogen peroxide; HRP: Horseradish peroxidase; ICC: Immunocytochemistry; LB: Lewy bodies; MALDI TOF: Matrix-assisted laser desorption/ionization time-offlight; MS: Mass spectrometry; MTS: 3-(4,5-dimethyl thiazol-2-yl)-5(3-carboxymethoxyphenyl)-2-(4-sulfophenyl)-2H-tetrazolium; m/z: Mass to charge ratio; PD: Parkinson's disease; PTM: Posttranslational modification; RNS: Reactive nitrogen species; ROS: Reactive oxygen species; RT: Room temperature; SDGC: Sucrose density gradient centrifugation; SEC: Size exclusion chromatography; SNCA: Alpha synuclein gene; TFA: Trifluoroacetic acid; ThT: Thioflavin T; TNM: Tetranitromethane; WB: Western blot; WT: Wild type.
\end{abstract}

\section{Competing interests}

The authors declare that they have no competing interests.

\section{Authors' contributions}

WX and SM designed the study, performed the experiments, analyzed the results, and wrote the manuscript; JCMS carried out cell culture experiments and was involved in drafting the manuscript; HM performed cell culture experiments; ACH performed Western blot analysis of aSyn and was involved in drafting the manuscript; USS carried out EM analysis and was involved in drafting the manuscript; CMB and JW contributed to the design of the study, and drafting the manuscript; JK designed the study and was involved in analysis and interpretation of data, and drafting the manuscript. All authors read and approved the final manuscript.

\section{Acknowledgements}

This study was supported by the grant of the Interdisciplinary Center for Clinical Research of the University Hospital, Erlangen, Germany (IZKF No. E11, E12, and E18) and the Bavarian State Ministry of Education and Culture, Science and Arts (ForlPS). Ingmar Henz, Annette Serwotka, Petra Wenzeler, and Rosa Weber are gratefully acknowledged for their excellent technical assistance.

\section{Author details \\ ${ }^{1}$ Institute of Biochemistry (Emil-Fischer-Center), Friedrich-Alexander-University of Erlangen-Nürnberg (FAU), Fahrstraße 17, 91054 Erlangen, Germany. ${ }^{2}$ Department of Molecular Neurology, University Hospital Erlangen, Friedrich-Alexander-Universität Erlangen-Nürnberg (FAU), 91054 Erlangen, Germany. ${ }^{3}$ Department of Ophthalmology, University Hospital Erlangen, Friedrich-Alexander-Universität Erlangen-Nürnberg (FAU), 91054 Erlangen, Germany.}

Received: 26 August 2014 Accepted: 9 February 2015

Published online: 11 March 2015

\section{References}

1. Baba M, Nakajo S, Tu PH, Tomita T, Nakaya K, Lee VM, et al. Aggregation of alpha-synuclein in Lewy bodies of sporadic Parkinson's disease and dementia with Lewy bodies. Am J Pathol. 1998;152(4):879-84.

2. Malkus KA, Tsika E, Ischiropoulos H. Oxidative modifications, mitochondrial dysfunction, and impaired protein degradation in Parkinson's disease: how neurons are lost in the Bermuda triangle. Mol Neurodegener. 2009;4:24.

3. Corti O, Lesage S, Brice A. What genetics tells us about the causes and mechanisms of Parkinson's disease. Physiol Rev. 2011;91(4):1161-218.

4. Polymeropoulos MH, Lavedan C, Leroy E, Ide SE, Dehejia A, Dutra A, et al. Mutation in the alpha-synuclein gene identified in families with Parkinson's disease. Science. 1997;276(5321):2045-7.

5. Kruger R, Kuhn W, Muller T, Woitalla D, Graeber M, Kosel S, et al. Ala30Pro mutation in the gene encoding alpha-synuclein in Parkinson's disease. Nat Genet. 1998;18(2):106-8.

6. Zarranz JJ, Alegre J, Gomez-Esteban JC, Lezcano E, Ros R, Ampuero I, et al. The new mutation, E46K, of alpha-synuclein causes Parkinson and Lewy body dementia. Ann Neurol. 2004;55(2):164-73.

7. Appel-Cresswell S, Vilarino-Guell C, Encarnacion M, Sherman H, Yu I, Shah B, et al. Alpha-synuclein p.H50Q, a novel pathogenic mutation for Parkinson's disease. Mov Disord. 2013;28(6):811-3.

8. Kiely AP, Asi YT, Kara E, Limousin P, Ling H, Lewis $P$, et al. alphaSynucleinopathy associated with G51D SNCA mutation: a link between Parkinson's disease and multiple system atrophy? Acta Neuropathol. 2013;125(5):753-69.

9. Proukakis C, Dudzik CG, Brier T, MacKay DS, Cooper JM, Millhauser GL, et al. A novel alpha-synuclein missense mutation in Parkinson disease. Neurology. 2012;80(11):1062-4.

10. Singleton $A B$, Farrer $M$, Johnson J, Singleton $A$, Hague $S$, Kachergus J, et al. alpha-Synuclein locus triplication causes Parkinson's disease. Science. 2003;302(5646):841.

11. Chartier-Harlin MC, Kachergus J, Roumier C, Mouroux V, Douay X, Lincoln S, et al. Alpha-synuclein locus duplication as a cause of familial Parkinson's disease. Lancet. 2004;364(9440):1167-9.

12. Ibanez P, Bonnet AM, Debarges B, Lohmann E, Tison F, Pollak P, et al. Causal relation between alpha-synuclein gene duplication and familial Parkinson's disease. Lancet. 2004;364(9440):1169-71.

13. Mizuta I, Satake W, Nakabayashi Y, Ito C, Suzuki S, Momose Y, et al. Multiple candidate gene analysis identifies alpha-synuclein as a susceptibility gene for sporadic Parkinson's disease. Hum Mol Genet. 2006;15(7):1151-8.

14. Brockmann K, Schulte C, Hauser AK, Lichtner P, Huber H, Maetzler W, et al. SNCA: major genetic modifier of age at onset of Parkinson's disease. Mov Disord. 2013;28(9):1217-21

15. Oueslati A, Fournier M, Lashuel HA. Role of post-translational modifications in modulating the structure, function and toxicity of alpha-synuclein: implications for Parkinson's disease pathogenesis and therapies. Prog Brain Res. 2010;183:115-45.

16. Waxman EA, Giasson BI. Molecular mechanisms of alpha-synuclein neurodegeneration. Biochim Biophys Acta. 2009;1792(7):616-24.

17. Vilar M, Chou HT, Luhrs T, Maji SK, Riek-Loher D, Verel R, et al. The fold of alpha-synuclein fibrils. Proc Natl Acad Sci U S A. 2008;105(25):8637-42.

18. Uchida K. 4-Hydroxy-2-nonenal: a product and mediator of oxidative stress. Prog Lipid Res. 2003:42(4):318-43.

19. Xiang W, Schlachetzki JC, Helling S, Bussmann JC, Berlinghof M, Schaffer TE, et al. Oxidative stress-induced posttranslational modifications of alpha-synuclein: specific modification of alpha-synuclein by 4-hydroxy-2nonenal increases dopaminergic toxicity. Mol Cell Neurosci. 2013:54:71-83.

20. Nasstrom T, Fagerqvist T, Barbu M, Karlsson M, Nikolajeff F, Kasrayan A, et al. The lipid peroxidation products 4-oxo-2-nonenal and 4-hydroxy-2-nonenal promote the formation of alpha-synuclein oligomers with distinct biochemical, morphological, and functional properties. Free Radic Biol Med. 2010;50(3):428-37.

21. Qin Z, Hu D, Han S, Reaney SH, Di Monte DA, Fink AL. Effect of 4-hydroxy-2-nonenal modification on alpha-synuclein aggregation. J Biol Chem. 2007;282(8):5862-70

22. Bae EJ, Ho DH, Park E, Jung JW, Cho K, Hong JH, et al. Lipid peroxidation product 4-hydroxy-2-nonenal promotes seeding-capable oligomer formation and cell-to-cell transfer of alpha-synuclein. Antioxid Redox Signal. 2013;18(7):770-83.

23. Esterbauer $\mathrm{H}$, Schaur RJ, Zollner $\mathrm{H}$. Chemistry and biochemistry of 4-hydroxynonenal, malonaldehyde and related aldehydes. Free Radic Biol Med. 1991;11(1):81-128. 
24. Yoritaka A, Hattori N, Uchida K, Tanaka M, Stadtman ER, Mizuno Y. Immunohistochemical detection of 4-hydroxynonenal protein adducts in Parkinson disease. Proc Natl Acad Sci U S A. 1996;93(7):2696-701.

25. Dalfo E, Portero-Otin M, Ayala V, Martinez A, Pamplona R, Ferrer I. Evidence of oxidative stress in the neocortex in incidental Lewy body disease. J Neuropathol Exp Neurol. 2005;64(9):816-30.

26. Castellani RJ, Perry G, Siedlak SL, Nunomura A, Shimohama S, Zhang J, et al. Hydroxynonenal adducts indicate a role for lipid peroxidation in neocortical and brainstem Lewy bodies in humans. Neurosci Lett. 2002;319(1):25-8.

27. Rutherford NJ, Moore BD, Golde TE, Giasson BI. Divergent effects of the H50Q and G51D SNCA mutations on the aggregation of alpha-synuclein. J Neurochem. 2014;131(6):859-67.

28. Khalaf O, Fauvet B, Oueslati A, Dikiy I, Mahul-Mellier AL, Ruggeri FS, et al. The H50Q mutation enhances alpha-synuclein aggregation, secretion and toxicity. J Biol Chem. 2014:289(32):21856-76.

29. Ghosh D, Mondal M, Mohite GM, Singh PK, Ranjan P, Anoop A, et al. The Parkinson's disease-associated H50Q mutation accelerates alpha-Synuclein aggregation in vitro. Biochemistry. 2013;52(40):6925-7.

30. Chi YC, Armstrong GS, Jones DN, Eisenmesser EZ, Liu CW. Residue histidine 50 plays a Key role in protecting alpha-synuclein from aggregation at physiological pH. J Biol Chem. 2014;289(22):15474-81.

31. Winner B, Jappelli R, Maji SK, Desplats PA, Boyer L, Aigner S, et al. In vivo demonstration that alpha-synuclein oligomers are toxic. Proc Natl Acad Sci U S A. 2011;108(10):4194-9.

32. Karpinar DP, Balija MB, Kugler S, Opazo F, Rezaei-Ghaleh N, Wender N, et al. Pre-fibrillar alpha-synuclein variants with impaired beta-structure increase neurotoxicity in Parkinson's disease models. Embo J. 2009;28(20):3256-68.

33. Poehler AM, Xiang W, Spitzer P, May V, Meixner H, Rockenstein E, et al. Autophagy modulates SNCA/alpha-synuclein release, thereby generating a hostile microenvironment. Autophagy. 2014;10(12):2171-92.

34. Lazaro DF, Rodrigues EF, Langohr R, Shahpasandzadeh H, Ribeiro T, Guerreiro P, et al. Systematic comparison of the effects of alpha-synuclein mutations on its oligomerization and aggregation. PLoS Genet. 2014;10(11):e1004741.

35. Klucken J, Poehler AM, Ebrahimi-Fakhari D, Schneider J, Nuber S, Rockenstein $\mathrm{E}$, et al. Alpha-synuclein aggregation involves a bafilomycin $\mathrm{A}$ 1-sensitive autophagy pathway. Autophagy. 2012;8(5):754-66.

36. Breitinger $U$, Breitinger $H G$, Bauer F, Fahmy K, Glockenhammer D, Becker CM. Conserved high affinity ligand binding and membrane association in the native and refolded extracellular domain of the human glycine receptor alpha1-subunit. J Biol Chem. 2004;279(3):1627-36.

\section{Submit your next manuscript to BioMed Central and take full advantage of:}

- Convenient online submission

- Thorough peer review

- No space constraints or color figure charges

- Immediate publication on acceptance

- Inclusion in PubMed, CAS, Scopus and Google Scholar

- Research which is freely available for redistribution

Submit your manuscript at www.biomedcentral.com/submit 